\title{
Comparação de Dois Métodos de Mensuração da Pressão Inspiratória Máxima em Pacientes Com e Sem Alterações do Nível de Consciência*
}

\author{
Comparison of Two Methods for Measurement of Maximal Inspiratory \\ Pressure in Patients with and without Alterations of the Conscience's Level
}

\author{
Ludmila Soccio Monteiro', Cristina Aparecida Veloso², Sebastião Araújo ${ }^{3}$, Renato Giovanni Giuseppe Terzi ${ }^{4}$
}

\section{RESUMO}

JUSTIFICATIVA E OBJETIVOS: Não existe consenso na literatura sobre o tempo de oclusão das vias aéreas necessário para se obter uma $\mathrm{PI}_{\max }$ verdadeira durante o desmame da ventilação mecânica (VM). Assim, o presente estudo teve por objetivos comparar dois métodos de mensuração da $\mathrm{PI}_{\max }$ e avaliar a influência do nível de consciência do paciente nessas medidas.

MÉTODO: População composta de 28 pacientes gerais de UTI, com tempo de VM $\geq 48 \mathrm{~h}$, em processo de desmame, divididos em dois grupos de acordo com a pontuação na escala de coma de Glasgow (ECGL): com (ECGL< $15)$ e sem alteração do nível de consciência (ECGL = 15). A via aérea foi ocluída com uma válvula unidirecional por 20s $\left(\mathrm{Pl}_{\max } \mathrm{T}_{20}\right)$, ou pelo tempo máximo de um minuto, se

1. Fisioterapeuta da Autarquia Hospitalar Municipal Regional do Campo Limpo - SP. Aluna de Mestrado, Departamento de Cirurgia da FCM-UNICAMP.

2. Fisioterapeuta Supervisora do Curso de Aprimoramento em Fisioterapia Respiratória em UTI do HC - UNICAMP. Docente do Curso de Graduação em Fisioterapia da UNIARARAS.

3. Professor Assistente Doutor do Departamento de Cirurgia da FCM - UNICAMP.

4. Professor Titular do Departamento de Cirurgia da FCM - UNICAMP.

*Recebido da Unidade de Terapia Intensiva do Hospital de Clínicas da Universidade Estadual de Campinas (HC - UNICAMP), Campinas, SP.

Apresentado em 03 de julho de 2006

Aceito para publicação em 05 de setembro de 2006

Endereço para correspondência:

Ludmila Soccio Monteiro

Rua Três de Maio, 82/22

Vila Clementino

04044-020 São Paulo, SP

Fone (11) 5084 1953/ (11) 72991716

E-mail: ludmilasm@yahoo.com.br

(C)Associação de Medicina Intensiva Brasileira, 2006 um platô de pressão inspiratória não foi observado durante três inspirações consecutivas $\left(\mathrm{PI}_{\max } \mathrm{T}_{\text {id }}\right)$.

RESULTADOS: $\mathrm{A} \mathrm{PI}{ }_{\operatorname{Max}} \mathrm{T}_{20}$ (média $\pm \mathrm{DP}, \mathrm{cmH}_{2} \mathrm{O}$ ) foi semelhante em ambos os grupos ( $44 \pm 16$ vs $42 \pm 15$, $p$ $=0,52)$. No entanto, os valores da $\mathrm{PI}_{\max } \mathrm{T}_{\mathrm{id}}$, assim como o tempo necessário para sua obtenção, foram maiores no grupo ECGL $<15\left(65 \pm 24\right.$ vs $47 \pm 23 \mathrm{cmH}_{2} \mathrm{O}$ e $37 \pm$ 10 vs $24 \pm 8 s, p=0,04$ e 0,0019, respectivamente).

CONCLUSÕES: O método comumente utilizado de 20s de oclusão da via aérea parece ser insuficiente para se mensurar a verdadeira $\mathrm{PI}_{\max }$ em pacientes com alterações do nível de consciência. Estudos adicionais, agora num grupo mais homogêneo de pacientes (p. ex.: com alterações neurológicas estruturais), são necessários para maior esclarecimento destes resultados.

Unitermos: alterações do nível de consciência, desmame, pressão inspiratória máxima, válvula unidirecional, ventilação mecânica.

\section{SUMMARY}

BACKGROUND AND OBJECTIVES: There is no literature consensus about the time of airway occlusion sufficient enough to get a true $\mathrm{PI}_{\max }$ during weaning from mechanical ventilation (MV). So, the main objectives of the present study were to compare two methods $\mathrm{PI}_{\max }$ measurement and to evaluate the influence of patients' level of conscience on them.

METHODS: The population was composed by $28 \mathrm{ge}-$ neral ICU patients, with $\mathrm{MV} \geq 48 \mathrm{~h}$, in a weaning process, divided into two groups according to Glasgow coma scale score: with (GCS < 15) and without (GCS $=15$ ) alterations of conscience level. The airway was occluded by using an unidirectional valve for 20s (PI$\max _{20}$ ), or for a maximum time of one minute if a plateau of inspiratory pressure was not observed during three consecutive inspirations $\left(\mathrm{PI}_{\max } \mathrm{T}_{\text {id }}\right)$. 
RESULTS: $\mathrm{PI}_{\max } \mathrm{T}_{20}$ (mean $\pm \mathrm{SD}, \mathrm{cmH}_{2} \mathrm{O}$ ) values were similar in both groups ( $44 \pm 16$ vs $42 \pm 15, \mathrm{p}=0.52)$. However, $\mathrm{PI}_{\max } \mathrm{T}_{\text {id }}$ values, as long as the time needed to their attainment, were greater in GCS $<15$ group $(65 \pm$ 24 vs $47 \pm 23 \mathrm{cmH}_{2} \mathrm{O}$ and $37 \pm 10$ vs $24 \pm 8 \mathrm{~s}, \mathrm{p}=0.04$ and 0.0019 , respectively).

CONCLUSIONS: The method commonly used of 20 s airway occlusion seems to be inadequate to get the true $\mathrm{PI}_{\max }$ in patients with alterations of the level of conscience. Additional studies, now in a more homogeneous group (e. g.: patients with structural brain lesion), are needed to clarify these findings.

Key Words: consciousness disturbances, maximum inspiratory pressure, mechanical ventilation, unidirectional valve, weaning.

\section{INTRODUÇÃO}

As mensurações da pressão inspiratória máxima $\left(\mathrm{PI}_{\max }\right)$ são consideradas estimativas reais da força muscular inspiratória. Pessoas normais bem motivadas e pacientes ambulatoriais estáveis, portadores de doenças pulmonares, são capazes de ativar maximamente ou próximo do máximo seus músculos respiratórios durante a manobra de aferição da $\mathrm{PI}_{\max }{ }^{1,2}$. Entretanto, não é comum que pacientes graves e dependentes de ventilação mecânica sejam capazes de ativar de forma máxima seus músculos inspiratórios durante esta manobra, porque podem não assimilar bem as instruções, podem estar sem energia ou não cooperativos. Assim, a mensuração da $\mathrm{PI}_{\max }$ pode subestimar consideravelmente a força muscular em alguns pacientes ${ }^{3}$.

Embora a $\mathrm{PI}_{\max }$ em pacientes graves continue sendo usada como um indicador global da função da musculatura respiratória, esta é altamente dependente de numerosas variáveis, que podem ser especialmente difíceis de controlar num ambiente de UTI. Baixos valores (p. ex.: -15 $\mathrm{cmH}_{2} \mathrm{O}$ ) não são comumente associados com o sucesso do desmame da ventilação mecânica, mas valores superiores a $-40 \mathrm{cmH}_{2} \mathrm{O}$ são freqüentemente preditivos do mesmo. Devido a esse questionável valor de predição, muitos o usam mais para seguir uma diretriz protocolar geral, do que como parte definitiva de uma série de critérios ${ }^{4}$.

O método mais comum para mensurar a $\mathrm{PI}_{\max }$ é baseado na oclusão do fluxo de ar após uma expiração forçada, ou seja, a partir do volume residual, sendo geralmente considerado o maior valor de três medidas consecutivas $^{5-8}$. Porém, acredita-se que o método descrito por Marini e col. ${ }^{9}$, onde a $\mathrm{PI}_{\max }$ foi mensurada com uma válvula unidirecional para permitir seletivamente a exalação enquanto a inspiração é bloqueada, é o mais indicado para ser utilizado em pacientes submetidos à ventilação mecânica ${ }^{10}$. Isso causa nos pacientes a iniciativa de sucessivos esforços de volumes respiratórios cada vez menores até atingir o volume residual, um fator conhecido que ajuda a gerar uma pressão mais negativa, dependendo, assim, mais da resposta fisiológica do paciente do que da sua colaboração.

De fato, num estudo prévio de Monteiro e col. ${ }^{11}$, num grupo heterogêneo de pacientes em desmame da ventilação mecânica, o tempo de oclusão das vias aéreas de 20 segundos, comumente utilizados na prática clínica, pareceu insuficiente para se atingir a $\mathrm{PI}_{\max }$ real, sugerindo que a mensuração da $\mathrm{PI}_{\max }$ com válvula unidirecional, com um valor platô de pressão negativa observado após três inspirações consecutivas, porém sem ultrapassar o tempo de um minuto de oclusão do fluxo aéreo, apresente valores mais reais que aqueles obtidos em tempo padrão pré-estabelecido, especialmente naqueles pacientes com algum grau de alteração do nível de consciência.

Assim, o presente estudo é uma complementação do anterior $^{11}$, em que foram comparados dois métodos de mensuração da $\mathrm{PI}_{\max }$ em pacientes em processo de desmame da ventilação mecânica, tendo agora por objetivo principal avaliar se o nível de consciência dos pacientes interfere nas medidas.

\section{MÉTODO}

A realização do presente estudo foi aprovada pelo Comitê Institucional de Ética em Pesquisa sob parecer $n^{\circ}$ 303/2003.

A população descrita no estudo anterior ${ }^{11}$, que consistiu numa amostra de conveniência, foi ampliada para 28 pacientes, de ambos os sexos, com idade $\geq 18$ anos, internados na Unidade de Terapia Intensiva (UTI) do Hospital das Clínicas da Universidade Estadual de Campinas (HC/UNICAMP), estáveis hemodinamicamente, em ventilação mecânica (VM) por tempo $\geq 48$ horas, sem uso de sedativos (exceto analgésicos) há pelo menos 24 horas e em processo de desmame da VM, de acordo com os seguintes critérios: ventilação espontânea, ventilação com pressão de suporte (VPS) de $10 \mathrm{cmH}_{2} \mathrm{O}$, pressão expiratória final (PEEP) de 5 $\mathrm{cmH}_{2} \mathrm{O}$ e fração inspirada de oxigênio $\left(\mathrm{FiO}_{2}\right) \leq 0,4$.

Inicialmente, o nível de consciência dos pacientes foi avaliado com a aplicação da escala de coma de Glasgow (ECGL), conforme sugerido por Knaus e col. ${ }^{12} \mathrm{e}$ 
Vincent e Berré ${ }^{13}$.

Em seguida, foi aplicado o método de aferição da PI-

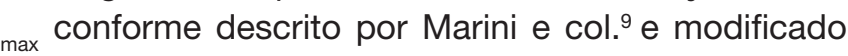
por Monteiro e col. ${ }^{11}$, pela oclusão da via inspiratória por tempos variáveis. O paciente foi colocado na posição Fowler $45^{\circ}$, sendo o procedimento esclarecido, quando consciente. Foi utilizado um manovacuômetro MV 300, da marca Ger-Ar, previamente calibrado, acoplado a uma válvula unidirecional através de peça em T. A higiene brônquica foi realizada 10 minutos antes da aplicação do protocolo e a freqüência cardíaca e a saturação periférica de oxigênio $\left(\mathrm{SpO}_{2}\right)$, foram monitorizadas e anotadas imediatamente antes e após o procedimento. As medidas foram realizadas por uma única pessoa e divididas em duas fases: a primeira, com um tempo de oclusão de 20 segundos $\left(T_{20}\right)$, e, a segunda, com um tempo de oclusão chamado "indeterminado", limitado por três inspirações consecutivas com um valor de platô de pressão negativa, sem, no entanto, ultrapassar um minuto de oclusão $\left(T_{\text {id }}\right)$. $A$ ordem de aplicação dos procedimentos foi aleatória, utilizando-se uma moeda (cara $-\mathrm{T}_{20}$ coroa $-\mathrm{T}_{\text {id }}$ ), com um intervalo de 15 minutos entre eles. Em cada fase foram realizadas três mensurações, com intervalo de um minuto entre elas, considerando-se, para efeitos de cálculos, o valor médio dessas três medidas.

\section{Análise Estatística}

A análise descritiva foi realizada por meio de medidas de posição e dispersão para variáveis contínuas e tabelas de freqüências para variáveis categóricas. Para comparação de proporções foi utilizado o teste Exato de Fisher e para comparação de medidas contínuas avaliadas em dois momentos na mesma unidade amostral foi aplicado o teste de Wilcoxon para amostras relacionadas.

Para comparação de medidas contínuas ou ordenáveis entre os dois grupos independentes foram aplicados os testes de Mann-Whitney e ANOVA (Análise de Variância) para medidas repetidas para comparação dos grupos. Foi aplicada a transformação por postos (ranks) nas medidas devido à variabilidade. O nível significativo adotado foi de $5 \%$.

\section{RESULTADOS}

As características demográficas, os diagnósticos principais, a pontuação na escala de coma de Glasgow (ECGL), o tempo de VM e os tipos de vias aéreas dos pacientes estão descritos na tabela 1. A idade média dos pacientes foi $52,9 \pm 16,6$ anos, sendo 23 do sexo masculino e cinco do sexo feminino, e o tempo médio de $\mathrm{VM}$ foi de $10,7 \pm 7,8$ dias.

Tabela 1 - Características Demográficas, Pontuação na Escala de Coma de Glasgow, Diagnósticos Principais, Tempo de Ventilação Mecânica e Tipos de Vias Aéreas dos Pacientes.

\begin{tabular}{|c|c|c|c|c|c|}
\hline Sexo & $\begin{array}{l}\text { Idade } \\
\text { (anos) }\end{array}$ & ECGL & Diagnóstico* & $\begin{array}{l}\text { Tempo } \\
\text { de VM } \\
\text { (dias) }\end{array}$ & $\begin{array}{c}\text { Tipo } \\
\text { de Via } \\
\text { Aérea** }^{*}\end{array}$ \\
\hline $\mathrm{M}$ & 45 & 6 & IAM/EAP & 6 & $\mathrm{TT}$ \\
\hline M & 55 & 10 & $\mathrm{AVCH}$ & 19 & TRAQ \\
\hline M & 20 & 11 & Politrauma/TCE & 6 & TT \\
\hline M & 73 & 10 & PO cirurgia cardíaca (RVM) & 3 & $\mathrm{TT}$ \\
\hline M & 20 & 15 & FAF/TRM (T4)/BCP & 13 & TRAQ \\
\hline M & 47 & 10 & Encefalopatia hepática & 3 & Tा \\
\hline $\mathrm{F}$ & 56 & 15 & IAM/EAP & 2 & Tा \\
\hline $\mathrm{F}$ & 49 & 15 & $\begin{array}{l}\text { Estado mal epiléptico/ } \\
\text { BCP }\end{array}$ & 19 & $\mathrm{TT}$ \\
\hline M & 76 & 15 & $\begin{array}{l}\text { PO esvaziamento cervical } \\
\text { (Ca epidermóide) }\end{array}$ & 3 & Tा \\
\hline M & 50 & 15 & $\begin{array}{l}\text { FAB toraco-abdminal/ } \\
\text { BCP/SDRA }\end{array}$ & 11 & TT \\
\hline M & 63 & 15 & PO cirurgia cardíaca (RVM) & 3 & TT \\
\hline $\mathrm{F}$ & 68 & 15 & PO cirurgia cardíaca (RVM) & 2 & $\pi$ \\
\hline M & 62 & 9 & $\begin{array}{l}\text { Politrauma/múltiplas fra- } \\
\text { turas }\end{array}$ & 16 & TRAQ \\
\hline $\mathrm{F}$ & 62 & 15 & $\begin{array}{l}\text { ICC/cardiopatia isquêmi- } \\
\text { ca/broncoespasmo }\end{array}$ & 29 & TRAQ \\
\hline M & 79 & 10 & $\begin{array}{l}\mathrm{PO} \text { retossigmoidectomia } \\
\text { (adenocarcinoma)/ TEP }\end{array}$ & 13 & Tा \\
\hline M & 65 & 15 & Tx hepático/BCP & 31 & TRAQ \\
\hline $\mathrm{F}$ & 64 & 15 & $\begin{array}{l}\text { PO esofagectomia (mega- } \\
\text { esôfago chagásico) }\end{array}$ & 3 & TT \\
\hline M & 59 & 6 & Tx hepático/encefalopatia & 19 & TRAQ \\
\hline M & 64 & 15 & PO neocistoplastia/sepse & 9 & TT \\
\hline M & 57 & 4 & $\begin{array}{l}\text { PO craniotomia base D } \\
\text { (Tu acústico) }\end{array}$ & 17 & TRAQ \\
\hline M & 43 & 6 & $\begin{array}{l}\text { TCE/craniotomia descom- } \\
\text { pressiva }\end{array}$ & 13 & TRAQ \\
\hline M & 52 & 15 & $\begin{array}{l}\text { PO esofagectomia (neo- } \\
\text { plasia)/BCP }\end{array}$ & 11 & TT \\
\hline M & 37 & 15 & Politrauma/BCP/SDRA & 13 & TRAQ \\
\hline M & 23 & 3 & $\begin{array}{l}\mathrm{PO} \text { ressecção total crane- } \\
\text { ofaringeoma }\end{array}$ & 7 & TRAQ \\
\hline M & 41 & 14 & TCE & 2 & $\mathrm{TT}$ \\
\hline M & 23 & 15 & TCE/contusão pulmonar & 11 & TRAQ \\
\hline M & 69 & 13 & IAM/choque cardiogênico & 10 & $\mathrm{TT}$ \\
\hline $\mathrm{M}$ & 58 & 6 & PO Tx renal/PCR & 5 & TT \\
\hline
\end{tabular}

* IAM: infarto agudo do miocárdio; EAP: edema agudo pulmonar; AVEH: acidente vascular encefálico hemorrágico; TCE: trauma craniencefálico; RVM: revascularização miocárdica; FAF: ferimento por arma de fogo; TRM: trauma raquimedular; $\mathrm{BCP}$ : broncopneumonia; $\mathrm{PO}$ : pós-operatório; Ca: câncer; $\mathrm{FAB}$ : ferimento por arma branca; SDRA: síndrome do desconforto respiratório agudo; ICC: insuficiência cardíaca congestiva; TEP: tromboembolismo pulmonar; Tx: transplante; D: direita; Tu: tumor; PCR: parada cardiorrespiratória.

** TT: tubo traqueal; TRAQ: traqueostomia. 
Os valores de $\mathrm{FC} \mathrm{e} \mathrm{SpO}_{2}$ obtidos antes das medidas de $\mathrm{PI}_{\max }$ pelos métodos $\mathrm{T}_{20}$ e $\mathrm{T}_{\text {id }}$ foram comparados, não se observando diferença estatística significativa entre eles, assegurando a homogeneidade dessas variáveis no momento da aplicação de cada método nos pacientes estudados (Tabela 2).

Tabela 2 - Análise Descritiva e Comparações das Medidas de FC (bpm) e $\mathrm{SpO}_{2}(\%)$ antes (A) da Mensuração da $\mathrm{PI}_{\text {máx }}$, no Grupo Total $(n=28)$.

\begin{tabular}{|c|c|c|c|c|c|c|}
\hline Variáveis & Média & DP & Mínimo & Mediana & Máximo & p-valor* \\
\hline $\mathrm{FC} \mathrm{T}_{20} \mathrm{~A}$ & 90,43 & 19,22 & 62,00 & 89,50 & 135,00 & \\
\hline $\mathrm{FC} \mathrm{T}_{\text {id }} \mathrm{A}$ & 91,04 & 20,60 & 60,00 & 88,00 & 138,00 & \\
\hline $\operatorname{Dif}(20$ - id $)$ & $-0,61$ & 6,71 & $-18,00$ & $-1,00$ & 13,00 & 0,6058 \\
\hline $\mathrm{SpO}_{2} \mathrm{~T}_{20} \mathrm{~A}$ & 97,18 & 2,36 & 92,00 & 97,50 & 100,00 & \\
\hline $\mathrm{SpO}_{2} \mathrm{~T}_{\text {id }} \mathrm{A}$ & 97,50 & 2,15 & 92,00 & 98,00 & 100,00 & \\
\hline $\operatorname{Dif}(20-$ id $)$ & $-0,32$ & 1,19 & $-4,00$ & 0,00 & 2,00 & 0,2100 \\
\hline
\end{tabular}

*Teste de Wilcoxon para amostras relacionadas

A média das medidas da $\mathrm{PI}_{\max } \mathrm{T}_{\text {id }}\left(55,8 \pm 25 \mathrm{cmH}_{2} \mathrm{O}\right)$ foi maior que a média das medidas da $\mathrm{PI}_{\max } \mathrm{T}_{20}$ $\left(43,1 \pm 15,5 \mathrm{cmH}_{2} \mathrm{O}\right)$ no grupo total $(\mathrm{n}=28)$, com $\mathrm{p}$ $=0,002$.

O tempo médio para a obtenção da $\mathrm{PI}_{\max } \top_{\text {id }}$, no grupo total $(n=28)$, foi de 30,2 $\pm 11,0$ segundos. Quando subdivididos em dois grupos, $\mathrm{ECGL}=15$ ( $\mathrm{n}=$ 14) e ECGL < $15(n=14)$, esse tempo médio foi de $23,8 \pm 7,7$ e $36,7 \pm 10$ segundos, respectivamente $(p=0,0019)$.

Quando foram fixados os métodos $\left(T_{20}\right.$ e $\left.T_{\text {id }}\right)$ e comparados os grupos (ECGL < 15 e ECGL $=15$ ), não foi observada diferença estatística significativa para a medida da $\mathrm{PI}_{\max }$ pelo método $\mathrm{T}_{20}(\mathrm{p}=0,5205)$, observando-se, porém, diferença significativa com o método $T_{\text {id }}(p=0,04)$. Quando se fixaram os grupos e comparam-se os métodos, a medida da $\mathrm{PI}_{\max }$ pelo método $T_{\text {id }}$ mostrou valor significativamente maior em relação ao método $T_{20}$ no grupo $E C G L<15(p=$ 0,0001), o mesmo não ocorrendo no grupo ECGL = $15(p=0,2153)$ (Tabela 3, Figura 1).

Tabela 3 - Valores da $\mathrm{PI}_{\max }\left(\mathrm{cmH}_{2} \mathrm{O}\right)$ pelos Métodos $\mathrm{T}_{20}$ e $\mathrm{T}_{\text {id }}$ nos Grupos ECGL < $15(n=14)$ e ECGL = $15(n=14)$.

\begin{tabular}{lllllll}
\hline Grupos & Variável & Média & DP & Mínimo & Mediana Máximo \\
\hline \multirow{2}{*}{ ECGL < 15 } & $\mathrm{PI}_{\text {max }} \mathrm{T}_{20}$ & 44,1 & 16,0 & 10,7 & 45,3 & 66,7 \\
& $\mathrm{PI}_{\max } \mathrm{T}_{\text {id }}$ & 64,8 & 24,1 & 25,3 & 69,3 & 106,7 \\
& $\mathrm{PI}_{\max } \mathrm{T}_{20}$ & 42,2 & 15,5 & 18,7 & 40,7 & 74,7 \\
\multirow{2}{*}{$\mathrm{ECGL}=15$} & $\mathrm{PI}_{\max } \mathrm{T}_{\text {id }}$ & 46,8 & 23,5 & 12,0 & 40,0 & 97,3 \\
\hline
\end{tabular}

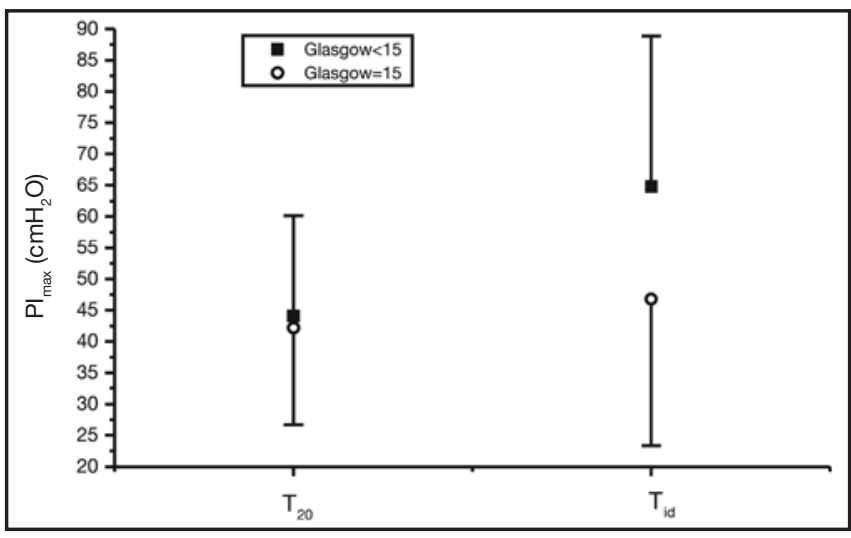

Figura 1 - Valores da $\mathrm{PI}_{\max }\left(\mathrm{cmH}_{2} \mathrm{O}\right)$ Obtidos pelos Métodos $\mathrm{T}_{20} \mathrm{e}$ $\mathrm{T}_{\text {id }}$, em Cada Grupo

Valores expressos em média \pm DP

( $\mathrm{p}$-valor grupo $=0,1415 ; \mathrm{p}$-valor método $<0,0001 ; \mathrm{p}$-valor método*grupo $=$ $0,0034)$.

\section{DISCUSSÃO}

Uma das principais metas de ordem prática por ocasião da elaboração do presente protocolo de pesquisa era a padronização da técnica de mensuração de $\mathrm{PI}_{\max }$ no serviço de fisioterapia respiratória da UTI-Adulto deste hospital, já que não existe um consenso na literatura, e mesmo dentro de serviços específicos, sobre a melhor maneira de sua aferição em pacientes submetidos à ventilação mecânica. De fato, é esperado que a padronização de uma determinada técnica e a sua incorporação como protocolo de aferição da $\mathrm{PI}_{\max }$ possibilitem uma uniformidade de atuação dos profissionais, favorecendo melhor análise e visão da evolução do paciente, optandose, então, pela sua mensuração com válvula unidirecional em dois tempos, com o intuito de torná-la rotina.

A padronização desta técnica pode trazer como benefícios aos pacientes, menor tempo de ventilação mecânica, devido a uma não subestimação da pressão inspiratória máxima ${ }^{14}$, e melhor avaliação da evolução do paciente em ventilação mecânica, especialmente durante o processo de desmame.

Marini e col..$^{9}$ descreveram um método em que a $\mathrm{PI}_{\max }$ foi mensurada com uma válvula unidirecional ocluída por 25 segundos (Método II), sendo este método comparado com o método da oclusão total de fluxo de ar no final da exalação (Método I) também pelo mesmo tempo. Os valores da $\mathrm{PI}_{\max }$ encontrados com válvula unidirecional quase invariavelmente excederam àqueles resultados sem o uso da válvula (média de variação $=34 \%, p<0,001$ ), presumivelmente porque volumes pulmonares menores melhoraram o desempenho dos músculos inspiratórios e aumentaram o esforço respiratório. Os valores de pico para ambos os 
métodos foram encontrados no período de 20 a 25 segundos em nove pacientes no método I e em 12 pacientes no método II. Este aumento no esforço respiratório pode ter sido causado pela inspiração bloqueada, portanto ineficaz para manter ventilação alveolar adequada ${ }^{15}$.

A mensuração da $\mathrm{PI}_{\max }$ com válvula unidirecional permite que mesmo os pacientes não colaborativos cheguem aproximadamente ao volume residual, aferindo-se, desse modo, a $\mathrm{Pl}_{\max }$ real. Porém, o tempo sugerido por Marini e col. ${ }^{9}$ pode subestimar os valores de $\mathrm{PI}_{\max }$ em alguns pacientes, como no caso daqueles que não se encontram colaborativos devido a um rebaixamento do nível de consciência, situação relativamente freqüente em pacientes de UTI, especialmente nos casos de pacientes com afecções neurológicas traumáticas ou clínicas.

Em pessoas normais, o estímulo químico da respiração aumenta exponencialmente com a duração da apnéia ${ }^{16}$. No método sem válvula unidirecional, ao contrário, o esforço respiratório é mais dependente da colaboração do que da resposta fisiológica do paciente. Outra razão possível para o aumento da $\mathrm{PI}_{\max }$ com a válvula unidirecional é que esta provavelmente permite a exalação até volumes pulmonares pequenos, próximos do volume residual, já descrito como um fator que aumenta o esforço respiratório ${ }^{17}$. De fato, Marini e col. ${ }^{9}$ têm sugerido que o estímulo do drive ventilatório deve ser a contribuição mais importante dos volumes pulmonares baixos, mais do que a posição do diafragma em si. Assim, espera-se que a $\mathrm{PI}_{\max }$ varie com a intensidade do drive ventilatório, com o volume pulmonar, com o tempo de oclusão e com a força da musculatura respiratória.

Marini e col. ${ }^{9}$ não observaram diferenças sistemáticas nos valores obtidos de $\mathrm{PI}_{\max }$ entre os métodos para os pacientes que puderam cooperar com a manobra e aqueles incapazes de fazê-lo $(p>0,05)$, tendo classificado como "alertas" os que responderam apropriadamente aos comandos verbais do pesquisador, e, os demais, confusos ou comatosos, foram classificados como "não alertas". No presente estudo utilizou-se a pontuação na ECGL para numerar este dado (nível de consciência), tentando deixá-lo menos subjetivo. Deste modo, pode ter acontecido que alguns pacientes considerados "alertas" no estudo de Marini e col. ${ }^{9}$ não tivessem de fato pontuação 15 na ECGL, dificultando assim a comparação.

Em pacientes gerais não neurológicos, a aplicação da ECGL para avaliar o nível de consciência pode ser questionada. Assim, em pacientes intubados, o componente verbal da ECGL não pode ser adequadamente avaliado, sendo em geral omitido porque o paciente não pode falar, e o escore é seguido pela letra $\mathrm{T}$ (de tubo traqueal). Ou- tros autores sugerem a utilização das recomendações do APACHE II ${ }^{12}$, em que um escore de um é dado quando se acredita que não teria nenhuma resposta verbal, um escore de cinco se uma resposta verbal normal é assumida, e um escore de três se a resposta verbal é questionável ${ }^{13}$. Esta é uma sugestão que foi adotada no presente estudo para se estimar o valor da pontuação na ECGL e, dessa forma, poder caracterizar numericamente o estado de consciência dos pacientes. No entanto, devido ao número relativamente pequeno de pacientes incluídos, optou-se pela divisão em apenas dois grupos: EGGL = 15 (pacientes totalmente alertas) e ECGL $<15$ (pacientes com algum grau de alteração do nível de consciência). Yamaguti e col. ${ }^{18}$ realizaram um estudo onde os objetivos foram comparar a $\mathrm{PI}_{\max }$ obtida pelo método convencional e pelo método da válvula unidirecional, em pacientes em processo de desmame de ventilação mecânica, e avaliar o possível efeito do aprendizado. $\mathrm{A} \mathrm{PI}_{\max }$ foi medida pelos dois métodos de forma aleatória, sendo realizadas 10 mensurações em cada um deles. Os maiores valores obtidos nas 10 medidas $\left(\mathrm{PI}_{\max 10}\right)$ e nas três primeiras medidas $\left(\mathrm{PI}_{\max 3}\right)$ de cada método foram selecionados para análise estatística. A PI ${ }_{\max }$ obtida pelo método convencional foi $48,9 \pm 18,4 \mathrm{cmH}_{2} \mathrm{O}$ e pela válvula unidirecional foi de $62 \pm$ $19,8 \mathrm{cmH}_{2} \mathrm{O}(\mathrm{p}<0,003)$. A PI $\max _{3}$ e $\mathrm{PI}_{\max 10}$ obtidas pelo método convencional foram, respectivamente, $45,1 \pm 16,4$ $\mathrm{cmH}_{2} \mathrm{O}$ e 48,9 $\pm 18,4 \mathrm{cmH}_{2} \mathrm{O}(\mathrm{p}<0,02)$, e pela da válvula unidirecional, $60,1 \pm 19,9 \mathrm{cmH}_{2} \mathrm{O}$ e $62 \pm 19,8 \mathrm{cmH}_{2} \mathrm{O}(\mathrm{p}=$ $0,06)$. Isto evidenciou que outras variáveis devem ser responsáveis por essa diferença, como o aumento do drive ventilatório decorrente de estímulos pulmonares mecânicos e metabólicos (retenção de dióxido de carbono), além da realização da manobra em volume pulmonar próximo ao volume residual, faz com que maiores valores sejam atingidos utilizando-se o método da válvula unidirecional, conforme discutido por Caruso e col. ${ }^{10}$.

Yamaguti e col. ${ }^{18}$ também concluíram que o método da válvula unidirecional é o mais adequado para mensuração da $\mathrm{PI}_{\max }$ em pacientes submetidos ao processo de desmame da ventilação mecânica invasiva, uma vez que produz maiores valores e não possui efeito aprendizado. Sendo assim, a realização de apenas três mensurações no método da válvula unidirecional foi suficiente para se obter o maior valor da $\mathrm{Pl}_{\max }$, e ainda afirmaram que os resultados desse estudo podem ser generalizados para todos os tipos de pacientes em processo de desmame da ventilação mecânica, inclusive os não colaborativos. Truwit e Marini ${ }^{19}$ realizaram um estudo que, segundo eles, permitiu mensuração acurada da $\mathrm{PI}_{\max }$ e com valores próximos do máximo, onde 22 pacientes críticos, intubados 
e cooperativos (habilidade de cooperação total avaliada pela administração de simples comandos e respostas a questões aritméticas simples) foram avaliados da seguinte maneira: nos primeiros 11 pacientes a $\mathrm{P}_{0,1}$ (pressão de oclusão mensurada durante os 100 milissegundos iniciais da inspiração) foi previamente mensurada, seguida da PI-

(válvula unidirecional ocluída por 20 a 25 segundos), mantendo o paciente alheio ao procedimento sendo apenas the informado que sentiria dificuldade para respirar. Após cinco minutos de descanso e explicações sobre o procedimento da mensuração da $\mathrm{PI}_{\max }$, esta foi novamente medida. Nos outros 11 pacientes, primeiro foi mensurada a $\mathrm{P}_{0,1}$, depois se adicionou na Unidade de Terapia Intensiva Cirúrgica um espaço morto de aproximadamente um terço do volume corrente que o ventilador mecânico estivesse ofertando ao circuito deste, e, após um ou dois minutos, foi mensurada novamente a $\mathrm{P}_{0,1}$, seguida da mensuração da $\mathrm{Pl}_{\max }$ sem explicação do procedimento. Após um descanso de cinco minutos e orientações quanto ao procedimento da mensuração da $\mathrm{PI}_{\max }$, esta foi novamente medida. Os resultados mostraram que houve diferença estatística significativa entre os 22 pacientes orientados e não orientados quanto ao procedimento de mensuração da $\mathrm{PI}_{\text {max }}$ ( $p$-valor $=0,001$ ). O p-valor, quando foram comparados os pacientes orientados com os não orientados que não utilizaram o espaço morto, foi de 0,005, e, para os pacientes orientados e não orientados que utilizaram o espaço morto este valor foi de 0,087 .

Os autores ${ }^{19}$ também observaram que pacientes com $\mathrm{P}_{0,1}$ $<2 \mathrm{cmH}_{2} \mathrm{O}$ tiveram um $\mathrm{p}$-valor $=0,004$ quando se comparou o valor da $\mathrm{PI}_{\max }$ com o paciente não orientado quanto o procedimento em relação à mensuração após a explicação, enquanto que para os pacientes com drives ventilatórios mais altos este valor foi de 0,097 . Nos pacientes em que o espaço morto foi adicionado demonstrou-se uma menor, mas não significativa estatisticamente, porcentagem de aumento com a orientação do procedimento, quando comparados com aqueles que não receberam o espaço morto ( $p$-valor $=0,071)$. A porcentagem de meIhora na $\mathrm{PI}_{\max }$ com a orientação foi significativa para os pacientes com $\mathrm{P}_{0,1}>2 \mathrm{cmH}_{2} \mathrm{O}$ versus aqueles com valores menores ou iguais a $2 \mathrm{cmH}_{2} \mathrm{O}(p$-valor $=0,013)$.

A orientação alterou significativamente os valores da $\mathrm{PI}_{\text {max }}$ apenas para os que tinham drives ventilatórios menores antes da mensuração, mas não para os pacientes com valores de $\mathrm{P}_{0,1}>2 \mathrm{cmH}_{2} \mathrm{O}$. Desse modo, mostraram que o uso do espaço morto substitui a necessidade de orientação do paciente quanto ao procedimento empregado para se chegar a valores maiores, podendo também utilizá-lo em pacientes com déficit no nível de consciência.
Assim, afirmaram que a oclusão da válvula unidirecional por 2 a 25 segundos produz aproximadamente a mesma $\mathrm{PI}_{\max }$ que a mensurada em pacientes graves e completamente cooperativos, se existe um drive ventilatório suficiente $\left(\mathrm{P}_{0,1}>2 \mathrm{cmH}_{2} \mathrm{O}\right)$ anterior à oclusão. Se a $\mathrm{P}_{0,1}$ for menor que $2 \mathrm{cmH}_{2} \mathrm{O}$, o espaço morto pode ser adicionado para aumentar o drive ventilatório, desde que o estresse da possível asfixia passageiro possa ser tolerada. Também relataram que o drive poderia ser aumentado pela redução do suporte ventilatório do paciente, porém esta técnica poderia resultar em fadiga da musculatura respiratória, enquanto que adicionando o espaço morto do paciente ventilado no modo assistido-controlado aumentaria o drive enquanto permitia que todos os ciclos respiratórios fossem mecanicamente assistidos ${ }^{19}$.

Comparando as conclusões anteriores de Truwit e Marini ${ }^{19}$ com os resultados obtidos no presente estudo, podese observar que, de uma maneira diferente, através de um tempo maior de oclusão, também se aumentou o drive ventilatório. Porém, não se avaliou a $\mathrm{P}_{0,1}$. Eles também encontraram maiores valores de $\mathrm{PI}_{\max }$ quando se utilizou o espaço morto, porém sem valor estatisticamente significativo quando comparado aos que não usaram ( $p$ valor $=0,071)$. Também, no mesmo estudo, quando se comparou os pacientes orientados e os não orientados quanto ao procedimento, e que usaram o espaço morto, o p-valor encontrado foi de 0,087 , diferente do presente estudo, em que, quando se utilizou um tempo de oclusão da válvula unidirecional maior do que 20 segundos $\left(T_{i d}\right)$ para os pacientes com ECGL $<15$, o p-valor foi igual a 0,001 . Truwit e Marini ${ }^{19}$ questionaram a possibilidade da fadiga muscular, mas possivelmente ela pôde ser evitada por meio do tempo de descanso adequado que foi dado aos pacientes, neste ensaio clínico, para se prevenir fadiga de alta freqüência (esforços repetitivos).

Para a $\mathrm{PI}_{\max }$, como pode ser visualizado na figura 1, observa-se diferença estatisticamente significativa entre os grupos $(E C G L<15$ versus $E C G L=15)$ no $T_{\text {id }}$ ( $p$-valor = 0,04), e no grupo ECGL < 15 quando se compara o método $\left(T_{20}\right.$ versus $\left.T_{\text {id }}\right)$ ( $p$-valor $\left.=0,0001\right)$, mostrando que o grupo ECGL < 15 necessitou de tempo maior de oclusão $\left(T_{i d}\right)$ que o $E C G L=15$ para que se chegasse ao valor da $\mathrm{PI}_{\text {max }}$, comprovando a hipótese de que pacientes com comprometimento neurológico necessitam de um tempo de oclusão maior do que 20 segundos para que a verdadeira $\mathrm{PI}_{\max }$ seja alcançada.

O fato do grupo ECGL < 15 ser composto por indivíduos apenas do sexo masculino não parece não ter influenciado os resultados, já que não houve grande diferença entre os valores da $\mathrm{PI}_{\max } \mathrm{T}_{20}$ para este grupo e o grupo ECGL $=15$. 
Quando se avaliam as médias das $\mathrm{PI}_{\max }$ (Tabela 3), observa-se que o valor da $\mathrm{PI}_{\max } \mathrm{T}_{\text {id }}$ no grupo ECGL $<15$ foi $31,94 \%$ maior que a $\mathrm{PI}_{\max } \mathrm{T}_{20}$ neste mesmo grupo, 27,78\% maior que a $\mathrm{PI}_{\text {max }} \mathrm{T}_{\text {id }}$ no grupo $E C G L=15$, e $34,88 \%$ para a $\mathrm{PI}_{\max } \mathrm{T}_{20}$ deste mesmo grupo. Assim, a $\mathrm{PI}_{\max } \mathrm{T}_{\text {id }}$ no grupo ECGL $<15$ foi em média 31,53\% maior que todos os outros valores de $\mathrm{PI}_{\text {max }}$. Estes achados estão de acordo com os de Rochester ${ }^{20}$, que a $\mathrm{PI}_{\max }$ medida próxima ao VR tende a aumentar seu valor em aproximadamente $30 \%$. Desse modo, é possível que os 20 segundos preconizados por Marini e col. ${ }^{9}$ não permitiram que esta mensuração fosse feita próxima do volume residual. De fato, Clanton e $\mathrm{Diaz}^{4}$ afirmaram que se a $\mathrm{PI}_{\max }$ e a $\mathrm{PE}_{\max }$ forem baixas, como ocorre nos pacientes graves, isto pode ser devido ao fato dessas mensurações serem feitas freqüentemente próximas da CRF, onde as forças elásticas dos pulmões e da caixa torácica, que contribuem substancialmente para estes valores, são neutralizadas.

A média do $T_{\text {id }}$ para o grupo $E C G L=15$ foi de 23,8 segundos apenas, já que estes pacientes não toleravam tempo maior de oclusão, devido provavelmente ao nível de consciência íntegro, chegando mais rapidamente ao platô de três inspirações consecutivas máximas. Isto justifica o valor médio de $\mathrm{PI}_{\max } \top_{\text {id }}$ para o grupo $\mathrm{ECGL}=15$, não muito maior que as outras mensurações, enquanto que $\circ T_{\text {id }}$ médio do grupo ECGL < 15 foi de 36,7 segundos. Desse modo, ainda não está claro se existe a necessidade de maior tempo de oclusão para os pacientes com nível de consciência comprometido, já que os valores encontrados neste grupo heterogêneo de pacientes, com 20 segundos de oclusão, não se mostraram estatisticamente diferentes entre os dois grupos ( $E C G L<15$ e ECGL = 15), não permitindo afirmar com segurança que neste tempo chega-se a valores submáximos para os pacientes com ECGL $<15$. Os resultados obtidos no presente estudo permitiram concluir que: 1) $\mathrm{A} \mathrm{PI}_{\max } \mathrm{T}_{\text {id }}$, neste grupo heterogêneo de pacientes em desmame da ventilação mecânica, mostraram valores superiores àqueles da $\mathrm{PI}_{\max } \mathrm{T}_{20}$; 2) no grupo $E C G L<15$ a $\mathrm{PI}_{\max } \mathrm{T}_{\text {id }}$ foi em média maior para o grupo $E C G L=15(p=0,04)$, sugerindo que pacientes com alterações no nível de consciência dependem mais da resposta fisiológica do organismo do que da cooperação, necessitando de tempo maior de oclusão da válvula unidirecional para se atingir a $\mathrm{PI}_{\max }$ real.

Assim, com base nestes resultados, há necessidade de novos estudos, agora num grupo mais homogêneo de pacientes, como aqueles com lesões neurológicas estruturais traumáticas ou não-traumáticas, para se concluir em definitivo se pacientes com pontuação na ECGL $<15$ necessitam de tempo de oclusão maior do que 20 segundos para se chegar à verdadeira $\mathrm{PI}_{\max }$, ou se meramente $\mathrm{a} \mathrm{PI}_{\max }$ $\mathrm{T}_{\mathrm{id}}$ representa uma supervalorização deste parâmetro.

\section{AGRADECIMENTOS}

Agradecemos à equipe de fisioterapia da UTI-HC-UNICAMP pela inestimável contribuição na execução do presente estudo, à Cleide Aparecida Moreira Silva da Comissão de Pesquisa da FCM-UNICAMP pela realização da análise estatística, não nos esquecendo de agradecer também a todos pacientes e familiares que nos deram o consentimento para a coleta de dados nesta pesquisa.

\section{REFERÊNCIAS}

01. Gandevia SC, McKenzie DK, Plassman BL - Activation of human respiratory muscles during different voluntary maneuvers. J Physiol, 1990;428:387-403.

02. Similowski T, Yan S, Gauthier AP et al - Contractile properties of the human diaphragm during chronic hyperinflation. N Eng J Med, 1991;325:917-923.

03. Multz AS, Aldrich TK, Prezant DJ et al - Maximal inspiratory pressure is not a reliable test of inspiratory muscle strength in mechanically ventilated patients. Am Rev Respir Dis, 1990;142:529-532.

04. Clanton TL, Diaz PT - Clinical assessment of the respiratory muscles. Phys Ther, 1995;75:983-995.

05. Black LF, Hyatt RE - Maximal respiratory pressures: normal values and relationship to age and sex. Am Rev Respir Dis, 1969;99:696-702.

06. Black LF, Hyatt RE - Maximal static respiratory pressures in generalized neuromuscular disease. Am Rev Respir Dis, 1971;103:641-650.

07. Yang K, Tobin MJ - A prospective study of indexes predicting the outcome of trials of weaning from mechanical ventilation. $\mathrm{N}$ Engl $\mathrm{J}$ Med, 1991;324:1445-1450.

08. Fiastro JF, Habib MP, Shon BY et al - Comparison of standard weaning parameters and the mechanical work of breathing in mechanically ventilated patients. Chest, 1988;94:232-238.

09. Marini JJ, Smith TC, Lamb V - Estimation of inspiratory muscle strength in mechanically ventilated patients: measurement of maximal inspiratory pressure. J Crit Care, 1986;1:32-38.

10. Caruso P, Friedrich C, Denari SDC et al - The unidirectional valve is the best method to determine maximal inspiratory pressure during weaning. Chest, 1999;115:1096-1101

11. Monteiro LS, Veloso CA, Araújo S et al - Comparação de dois métodos de mensuração da pressão inspiratória máxima com o uso de uma válvula unidirecional. RBTI, 2004;16:74-77.

12. Knaus WA, Draper EA, Wagner DP et al - APACHE II: a severity of disease classification system. Crit Care Med, 1985;13:818-829.

13. Vincent $\mathrm{JL}$, Berre $\mathrm{J}$ - Primer on medical management of severe brain injury. Crit Care Med, 2005;33:1392-1399.

14. Kacmarek RM, Cycyk-Champman MC, Young-Palazzo PJ et al - Determination of maximal inspiratory pressure: a clinical study and literature review. Respir Care, 1989;34:868-878.

15. Sassoon CS, Te TT, Mahutte CK et al - Airway occlusion pressure. An important indicator for successful weaning in patients with chronic obstructive pulmonary disease. Am Rev Respir Dis, 1987;135:107-113.

16. Godfrey S, Campbell EJ - The control of breath holding. Respir Physiol, 1968;5:385-400.

17. Rahn $\mathrm{H}$, Otis $\mathrm{AB}$, Chadwick LE et al - The pressure-volume diagram of the thorax and lung. Am J Physiol, 1946;146:161-178.

18. Yamaguti WPS, Alves LA, Kauss IAM et al - Comparação entre a pressão inspiratória máxima medida pelo método da válvula unidirecional e pelo convencional em pacientes submetidos ao processo de desmame da ventilação mecânica invasiva. RBTI, 2004;16:142-145.

19. Truwit JD, Marini JJ - Validation of a technique to assess maximal inspiratory pressure in poorly cooperative patients. Chest, 1992;102:1216-1219.

20. Rochester DF - The diaphragm: contractile properties and fatigue. J Clin Invest, 1958;75:1397-1402. 